\title{
Molecular histology 2020: beyond what we can see
}

\author{
Ivelisse Sánchez Diaz ${ }^{1}$
}

Published online: 5 February 2020

(c) Springer Nature B.V. 2020

The growing focus on precision medicine and target-based treatments using compounds, biologicals or gene therapies, positions the Journal for Molecular Histology at the forefront with an opportunity to make an impactful contribution by publishing relevant research that may help guide effective approaches for the development and delivery of these treatments. For instance, the analysis of histological features of cells and tissues, continues to guide pathological diagnosis for many conditions. Understanding off-target and secondary effects of many drugs is aided by studies that define the differential distribution of receptors and other molecules throughout tissues and specific developmental stages. Furthermore, the design of genetic treatments requires a basic understanding of the tissue and cellular expression of the gene products, both RNA and protein, as well as their function. Technologies such as, Cryogenic electron microscopy, Synchrotron X-Ray imaging, Two-photon excitation microscopy, Magnetic resonance imaging and Computed Tomography, to name a few, open the door to discover new insights into the structure-function relationships at an ultra structural level in living tissues and how these are altered in pathologies. I believe that it is by placing our focus on key scientific questions that need to be addressed and using the technologies available as tools through collaborative efforts if needed, that we could obtain answers that are relevant and may have a positive impact on the health challenges that still face us.

My vision for this new phase of the Journal of Molecular Histology is to continue to offer a user-friendly platform for high-quality research and focus on attracting these contributions from the broadest spectrum of scientists who probe into the functional characterization of the different cells and

Ivelisse Sánchez Diaz

isanchez@igtp.cat; ivelisse.sanchez@icloud.com

1 Functional Biology and Experimental Therapeutics Laboratory, Neurogenetics Unit, Department of Neuroscience, Health Sciences Research Institute Germans Trias i Pujol (IGTP), Universitat Autónoma de Barcelona (UAB), Badalona, Barcelona, Spain tissues in normal and pathological conditions. To accomplish this we will take more advantage of web-based and social network resources to increase visibility of the Journal and potentiate the far-reaching impact that the quality of its content deserves. Going forward, we plan to publish issues focused on hot research topics in molecular histology of broad interest. Investigators who trust our Journal to bring forth their high-quality research to the general scientific community are important to us. Therefore, we will continue to pay especial attention to the efficient processing of submissions and time to publication as it is in the best interest of researchers and allows science to move forward. In addition, we will continue highlighting publications with demonstrated impact on the scientific community as shown by their citations. Moreover, a dedicated Editorial Board of scientists is of outmost importance in achieving all of these goals and we will continue to have a team, both experts in their field and committed to excellence in scientific publication and promoting science for good. This new team will be announced shortly. Of course, the comments and suggestions from insightful reviewers are most critical in all of these endeavors. Their compromise with the peer-review process to select high-quality research for publication in a fair and professional manner is highly valued. We are especially grateful for the Journal is well supported by an excellent team of dedicated Editorial staff professionals at Springer Nature who ensure the efficient and smooth every day functions of the Journal.

In closing, we would like to thank Professor Brian Key and his Editorial Board members for their dedicated work and commitment during these last years and look forward to their continued support and scientific contributions.

We hope to count on all previous contributors, friends and subscribers of the Journal of Molecular Histology as we move forward and into the new decade. 
*Ivelisse Sánchez Diaz, PhD. Editor-in-Chief of the Journal of Molecular Histology, Principal Investigator and co-Director of the Functional Biology and Experimental therapeutics lab at the Health Sciences Research Institute Germans Trias i Pujol (IGTP), Universitat Autónoma de Barcelona and co-founder of the academic spin-off Biointaxis, S.L, Spain.
Publisher's Note Springer Nature remains neutral with regard to jurisdictional claims in published maps and institutional affiliations. 